\title{
Change in product selectivity during the production of glyceric acid from glycerol by Gluconobacter strains in the presence of methanol
}

\author{
Shun Sato ${ }^{1}$, Naoki Morita ${ }^{2}$, Dai Kitamoto ${ }^{1}$, Toshiharu Yakushi ${ }^{3}$, Kazunobu Matsushita ${ }^{3}$ and Hiroshi Habe ${ }^{\text {* }}$
}

\begin{abstract}
To enhance the value-added use of methanol-containing raw glycerol derived from biodiesel fuel production, the effect of methanol supplementation on glyceric acid (GA) production by Gluconobacter spp. was investigated. We first conducted fed-batch fermentation with Gluconobacter frateurii NBRC103465 using raw glycerol as a feeding solution. GA productivity decreased with increasing dihydroxyacetone (DHA) formation when the raw glycerol contained methanol. The results of this experiment and comparative experiments using a synthetic solution modeled after the raw glycerol indicate that the presence of methanol caused a change in the concentrations of GA and DHA, two glycerol derivatives produced during fermentation. Other Gluconobacter spp. also decreased GA production in the presence of $1 \%(V / V)$ methanol. In addition, purified membrane-bound alcohol dehydrogenase $(m A D H)$ from Gluconobacter oxydans, which is a key enzyme in GA production, showed a decrease in dehydrogenase activity toward glycerol as the methanol concentration increased. These results strongly suggest that the observed decrease in GA production by Gluconobacter spp. resulted from the methanol-induced inhibition of mADH-mediated glycerol oxidation.
\end{abstract}

Keywords: Acetic acid bacteria, Glyceric acid, Methanol, Membrane-bound alcohol dehydrogenase, Raw glycerol

\section{Introduction}

Biodiesel fuel (BDF), a class of renewable energy, is widely used to conserve fossil fuels and reduce carbon dioxide emissions. One method of BDF production involves transesterification between triacylglycerols, which are present in plant oils and animal fats, and methanol under alkaline conditions. This reaction forms glycerol as a byproduct at $10 \%$ of the initial amount of triacylglycerol. Because BDF production has rapidly increased in the US and in European and Asian countries (Rahmat et al. 2010; Glycerin market report, 2011), residual glycerol waste (raw glycerol) is now being used in chemical industries as an alternative feedstock. Therefore, many research groups have developed chemical and biological techniques for the conversion of glycerol into value-added chemicals such as epichlorohydrine (Kubicek et al. 2005), 1,3-propanediol

\footnotetext{
* Correspondence: hiroshi.habe@aist.go.jp

'Research Institute for Innovation in Sustainable Chemistry, National Institute of Advanced Industrial Science and Technology (AIST), Tsukuba Central 5-2, 1-1-1 Higashi, Tsukuba, Ibaraki 305-8565, Japan

Full list of author information is available at the end of the article
}

(Rehman et al. 2008), 3-hydroxypropionic acid (Rathnasingh et al. 2009), and 2,3-dihydroxypropionic acid (glyceric acid, GA) (Habe et al. 2009a).

Raw glycerol derived from BDF production has diverse properties that depend on the initial raw materials (e.g., origin of triacylglycerol), reaction conditions, and manufacturing process. The transesterification of triacylglycerol with methanol requires excess methanol for efficient BDF production, resulting in an impure, methanol-containing raw glycerol. Although methanol recovery by evaporation during the BDF manufacturing process is relatively easy, it requires additional energy and is costly. Therefore, the use of raw glycerol is economically preferable, and so the technological utility of glycerol that contains impurities, such as methanol and alkali metals, should be developed. Indeed, raw glycerol after purification has relatively high purity and represents a useful raw material for chemical production, whereas impure raw glycerol is often wasted. Therefore, it is important to investigate the effects of impurities in raw glycerol, particularly methanol, on chemical production. 
Our recent research has focused on microbial GA production from glycerol and the applications of GA because of its simple but chiral structure, which provides building blocks for various fine chemicals (Habe et al. 2009b). GA itself has been reported to have biological activity, such as accelerating the oxidation of ethanol (Eriksson et al. 2007) and enhancing the viability of ethanol-dosed gastric cells (Habe et al. 2011a). Applications of GA as a value-added material, including as an antitrypsin compound (Habe et al. 2011b), a bioplastic monomer (Fukuoka et al. 2011), and novel surfactants (Fukuoka et al. 2012), have also been investigated. With regard to the production of GA, a biological method that involves the enantioselective conversion of glycerol to D-GA by acetic acid bacterial fermentation has been developed (Habe et al. 2009c). Gluconobacter frateurii NBRC103465 showed the highest GA productivity $(136 \mathrm{~g} / \mathrm{L})$, whereas Acetobacter tropicalis NBRC16470 produced D-GA with 99\% ee (Habe et al. 2009a).

Concerning the effect of raw glycerol containing methanol on GA production by acetic acid bacteria, we demonstrated that Gluconobacter sp. NBRC3259 produced less GA when raw glycerol from which impurities had not been removed was applied (Habe et al. 2009d). It was also shown that this strain produced only a small amount of GA in the presence of $1 \%(v / v)$ methanol. In contrast, we developed a fed-batch fermentation method that uses glycerol in an alkaline solution for glycerol feeding and $\mathrm{pH}$ control (Habe et al. 2009a). Because raw glycerol usually contains alkaline metals such as sodium and potassium, it is of interest to investigate the feasibility of utilizing raw glycerol in fed-batch fermentation.

In this study, we found that GA production decreased, whereas the production of dihydroxyacetone (DHA), a byproduct of GA fermentation (Figure 1), increased when we used raw glycerol as a feeding solution for fed-batch fermentation with G. frateurii NBRC103465. Hence, to elucidate the reason for the change in the glycerol derivatives produced, we investigated GA production by various Gluconobacter spp. and glycerol oxidation in the presence of methanol.

\section{Materials and methods}

\section{Bacterial strains and culture conditions}

Gluconobacter frateurii NBRC103465, G. frateurii THD32 (Toyama et al. 2005) and its $\Delta s l d A$ mutant (Toyama et al. 2005; Soemphol et al. 2008), and Gluconobacter oxydans IFO12528 and its $\triangle a d h A$ mutant (Habe et al. 2009a) were used for GA production. Seed cultures of the Gluconobacter strains were prepared in $5 \mathrm{~mL}$ of glucose medium composed of $5 \mathrm{~g} / \mathrm{L}$ glucose, $5 \mathrm{~g} / \mathrm{L}$ yeast extract, $5 \mathrm{~g} / \mathrm{L}$ polypepton, and $1 \mathrm{~g} / \mathrm{L}_{\mathrm{MgSO}_{4}}$. $7 \mathrm{H}_{2} \mathrm{O}$ at $30^{\circ} \mathrm{C}$ and $200 \mathrm{rpm}$ for $24 \mathrm{~h}$. The seed cultures $(1.5 \mathrm{~mL})$ were transferred to $300-\mathrm{mL}$ Erlenmeyer flaks containing $30 \mathrm{~mL}$ of glycerol medium composed of $170 \mathrm{~g} / \mathrm{L}$ glycerol, $10 \mathrm{~g} / \mathrm{L}$ polypepton, $1 \mathrm{~g} / \mathrm{L}$ yeast extract, $1 \mathrm{~g} / \mathrm{L} \mathrm{MgSO}_{4} \cdot 7 \mathrm{H}_{2} \mathrm{O}, 0.9 \mathrm{~g} / \mathrm{L} \mathrm{KH}_{2} \mathrm{PO}_{4}$, and $0.1 \mathrm{~g} / \mathrm{L} \mathrm{K}_{2} \mathrm{HPO}_{4}$. The cultures were incubated at $30^{\circ} \mathrm{C}$ and $200 \mathrm{rpm}$ on a rotary shaker for $96 \mathrm{~h}$. When needed, $50 \mathrm{mg} / \mathrm{L}$ kanamycin for the $\triangle a d h A$ strain of $\mathrm{G}$. oxydans and the $\Delta$ sldA strain of G. frateurii THD32 was added to the medium.

\section{Jar fermentation experiment}

Gluconobacter frateurii was cultured in a 5-L jar fermenter containing $2.5 \mathrm{~L}$ of glycerol medium (Habe et al. 2009a). The seed cultures $(90 \mathrm{~mL})$ were used for inoculation. Cultivation was done at $30^{\circ} \mathrm{C}, 500 \mathrm{rpm}$, and 0.5 $\mathrm{vvm}$, and maintained at $\mathrm{pH} 6$ using raw glycerol derived from BDF production or the model solution. The raw glycerol contained the following components: glycerol, 66.4\% (w/v); methanol, 30.9\% $(w / v)$; and sodium salt, $0.54 \%(w / v)$; pH 12 (Habe et al. 2009d). In contrast, based on the raw glycerol composition, a synthetic solution modeled after the raw glycerol was prepared with the following pure reagents: glycerol, 66\% $(w / v)$; methanol, $30 \%(w / v)$; and sodium hydroxide, $1 \mathrm{M}$.

\section{Analysis of the culture broth}

The glycerol, GA, and DHA concentrations in the culture broth were determined by high-performance liquid chromatography (HPLC), as described previously (Habe et al. 2009a). In addition, the methanol concentration was determined by HPLC using the same method as that employed for the GA assay.

Bacterial growth was evaluated by OD measurements at $600 \mathrm{~nm}$ using a V-530 UV/VIS spectrophotometer (JASCO Corp., Tokyo, Japan).

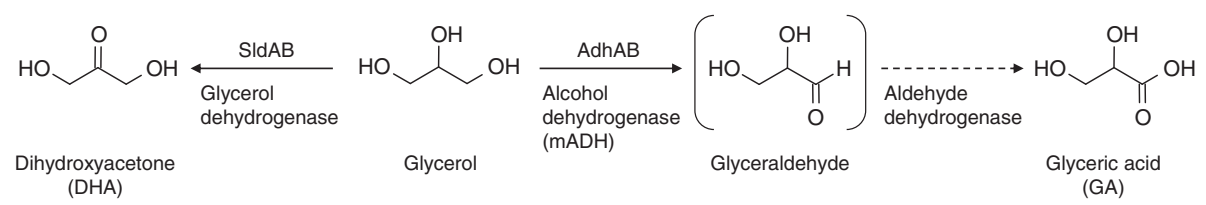

Figure 1 Pathways for GA and DHA formation from glycerol by Gluconobacter spp. The dashed arrow indicates an unidentified enzymatic reaction in GA formation. AdhAB, membrane-bound alcohol dehydrogenase, which is referred to as $m A D H$; SldAB, membrane-bound glycerol dehydrogenase, which is referred to as SLDH or GLDH. 


\section{Evaluation of the ability of $G$. oxydans and its $\triangle a d h A$ mutant to assimilate methanol}

Whole cells of G. oxydans IFO12528 and its $\triangle a d h A$ strain were used to investigate methanol consumption. Cells were precultured in $30 \mathrm{~mL}$ of glucose medium in $300-\mathrm{mL}$ Erlenmeyer flasks at $30^{\circ} \mathrm{C}$ and $200 \mathrm{rpm}$ for $24 \mathrm{~h}$. Cells collected from $60 \mathrm{~mL}$ of the culture by centrifugation were suspended in $30 \mathrm{~mL}$ of glycerol medium containing 0$10 \%(v / v)$ methanol. The initial $\mathrm{OD}_{600}$ was $\sim 2.0$. The flasks were shaken at $30^{\circ} \mathrm{C}$ and $200 \mathrm{rpm}$, and an aliquot of the broth was removed at regular intervals for HPLC.

\section{Enzyme assays}

Glycerol dehydrogenase activity in the presence of methanol was evaluated using purified, membrane-bound $G$. oxydans alcohol dehydrogenase (mADH) based on the method of Adachi et al. (1978) with modifications. Briefly, the reaction mixture contained $0.4 \mathrm{~mL}$ of McIlvaine buffer (pH 5.0), $10 \mathrm{mM}$ potassium ferricyanide, $8 \mathrm{mM}$ sodium azide, enzyme solution, 5-20\% ( $w / v)$ glycerol, and/or $0-2 \%$ $(w / v)$ methanol in a total volume of $1.0 \mathrm{~mL}$. The reaction was carried out at $25^{\circ} \mathrm{C}$ for $5 \mathrm{~min}$ by adding ferricyanide solution, and stopped by adding $0.5 \mathrm{~mL}$ of ferric sulfateDupanol reagent (Wood et al. 1962). The resulting mixture was allowed to stand at $25^{\circ} \mathrm{C}$ for $20 \mathrm{~min}$, and then the absorbance of the solution was measured at $660 \mathrm{~nm}$ to estimate the intensity of the Prussian blue color formed. The oxidation of $1 \mu \mathrm{mol}$ of substrate was equal to 4.0 absorbance units. One unit of dehydrogenase activity was defined as the amount of enzyme that catalyzed the oxidation of $1 \mu \mathrm{mol}$ of substrate in $1 \mathrm{~min}$. After the determination of dehydrogenase activity toward 1.2 and $2 \%(w / v)$ methanol, that towards $20 \%(w / v)$ glycerol in the presence of various methanol concentrations was calculated by subtracting the value of the activity toward methanol from that toward glycerol in the presence of methanol.

The protein concentration of the purified enzyme was determined by the Lowry method using bovine serum albumin as the standard.

\section{Results}

Fed-batch GA fermentation by G. frateurii NBRC103465 using raw glycerol derived from BDF production and its modeled solution

Previously, Habe et al. (2009a) demonstrated that fedbatch fermentation using glycerol in an alkaline feeding solution resulted in considerable improvement in GA production by $G$. frateurii NBRC103465, indicating a potential use for raw glycerol derived from BDF production, which contains both alkali metals and methanol. Therefore, we first attempted to use raw glycerol $(66.4 \%$ $[w / v]$ glycerol and 30.9\% [w/v] methanol, $\mathrm{pH} 12$; Habe et al. 2009d) as a feeding solution for GA fermentation by G. frateurii NBRC103465. As shown in Figure 2a, GA was barely detectable in the culture broth, whereas DHA was present at $>50 \mathrm{~g} / \mathrm{L}$ after 5 days of cultivation. In contrast, after the removal of methanol $(40 \%[w / v]$ glycerol), $81 \mathrm{~g} / \mathrm{L}$ GA and $38 \mathrm{~g} / \mathrm{L}$ DHA were produced after 5 days of cultivation, despite the presence of other impurities in the original glycerol (Figure 2b). This suggests that the addition of methanol along with raw glycerol to the culture altered the production of GA and DHA by G. frateurii. Note that glycerol content in the culture using raw glycerol containing methanol as a feeding solution slightly increased during the cultivation, though $51 \mathrm{~g} / \mathrm{L}$ of DHA was produced (Figure 2a). In this case, the feeding rate of glycerol accompanied with $\mathrm{pH}$ control exceeded the consumption rate of glycerol for cell growth and DHA production, resulting in a gradual accumulation of glycerol in the culture broth. In contrast, glycerol content in the culture using raw glycerol containing no methanol as a feeding solution decreased during the cultivation (Figure $2 \mathrm{~b}$ ), probably because a large amount of GA was produced.

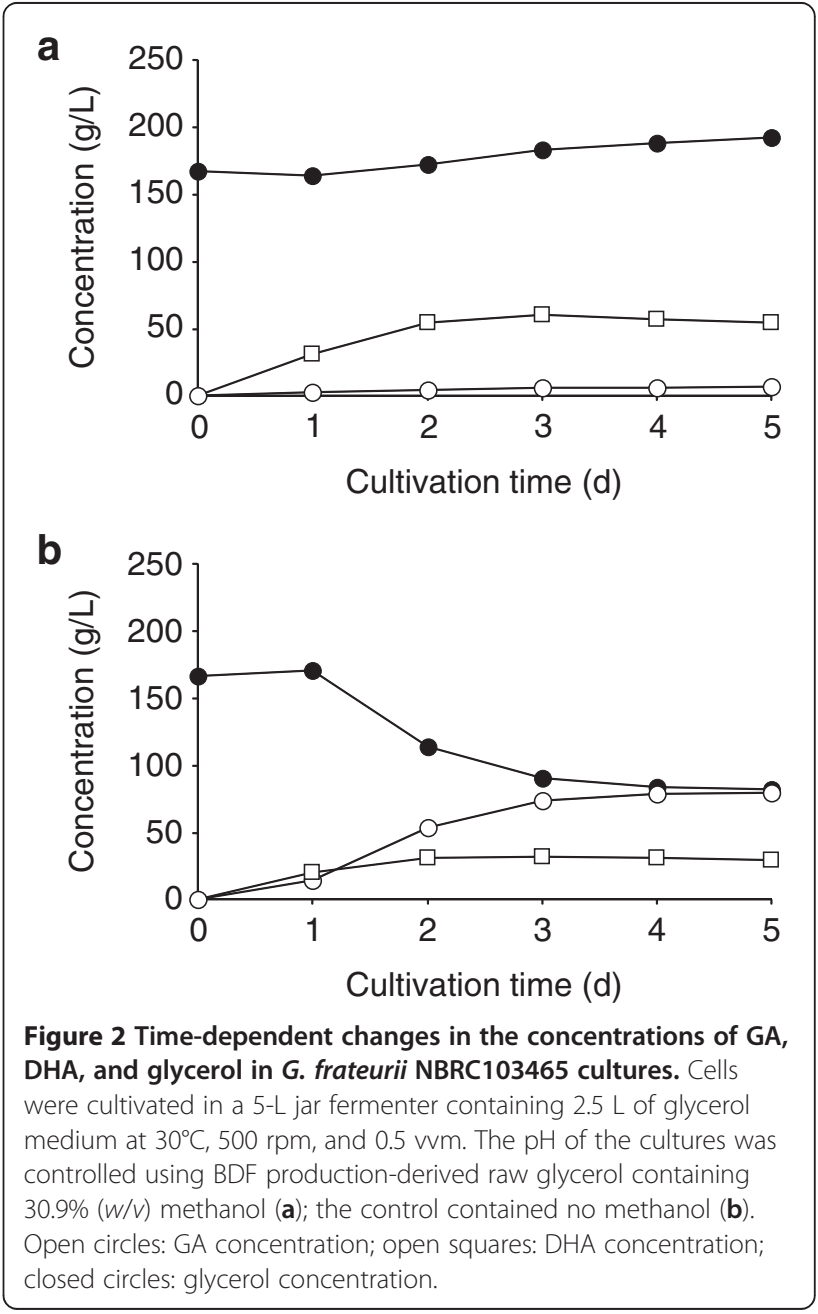


To clarify whether the above change in product selectivity was caused by methanol supplementation or by that in combination with other impurities in the raw glycerol (e.g., free fatty acids, methyl esters of fatty acids, and residual triacylglycerols), the same experiment was performed using a synthetic solution modeled after the raw glycerol. This solution consisted of $66 \%(w / v)$ glycerol and $30 \%(w / v)$ methanol in $1 \mathrm{M} \mathrm{NaOH}$; a methanol-free version of the solution was used as a control. Figure 3 shows the cell growth and GA and DHA production profiles of G. frateurii. Cell growth was unaffected, but GA and DHA production were altered by methanol supplementation. In the absence of methanol, GA predominated (73 g/L); only $20 \mathrm{~g} / \mathrm{L}$ DHA had been produced after $84 \mathrm{~h}$ of cultivation. In contrast, in the presence of methanol, DHA predominated $(>50 \mathrm{~g} / \mathrm{L})$ and the GA level was $<10 \mathrm{~g} / \mathrm{L}$. This result shows that methanol caused a change in product selectivity between GA and DHA with almost the same bacterial growth profile.

\section{GA production by Gluconobacter spp. in the presence of methanol}

To determine the effect of methanol on GA production by Gluconobacter strains other than G. frateurii NBRC103465, we examined GA and DHA production from glycerol in the presence of methanol by $G$. frateurii THD32 and G. oxydans IFO12528. The strains were cultivated in $300-\mathrm{mL}$ flasks containing $30 \mathrm{~mL}$ of glycerol medium. Table 1 summarizes the amounts of GA and DHA produced after $96 \mathrm{~h}$ of cultivation. All of

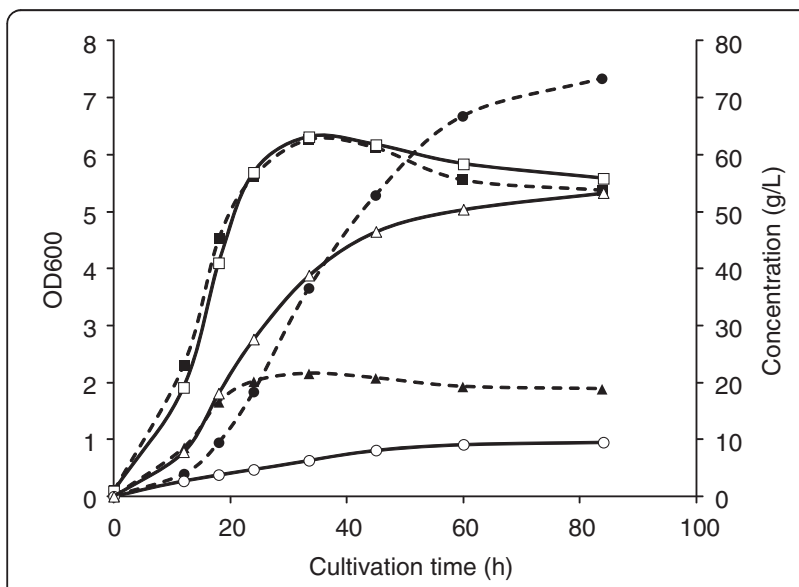

Figure 3 Time-dependent changes in cell growth and the concentrations of GA and DHA in G. frateurii NBRC103465 cultures. Cells were cultivated in a 5 - $\mathrm{L}$ jar fermenter containing $2.5 \mathrm{~L}$ of glycerol medium at $30^{\circ} \mathrm{C}, 500 \mathrm{rpm}$, and $0.5 \mathrm{vvm}$. The $\mathrm{pH}$ of the cultures was controlled using a synthetic solution modeled after the raw glycerol, which contains 30\% (w/V) methanol (solid lines, open symbols); the control contained no methanol (broken lines, closed symbols). Cell growth was evaluated by measurement of the $\mathrm{OD}$ at $600 \mathrm{~nm}$. Squares: cell growth; circles: GA concentration; triangles: DHA concentration. the strains tested produced less GA in the presence of $1 \%(v / v)$ methanol than with no methanol. In addition, the amount of DHA produced was increased in methanolsupplemented cultures of G. frateurii NBRC103465 and G. oxydans IFO12528, whereas methanol supplementation did not affect DHA production in the G. frateurii THD32 cultures. This result suggests that methanol decreases GA production by Gluconobacter spp.

\section{Effect of methanol supplementation on the G. oxydans $\triangle$ adhA mutant and purified $\mathrm{mADH}$}

We investigated the effect of methanol on the activity of a key enzyme in GA production, $\mathrm{mADH}$, which catalyzes the oxidation of glycerol to glyceraldehyde and is encoded by adhA (Habe et al. 2009a, 2010). This enzyme exhibits weak methanol dehydrogenase activity as compared to ethanol (Adachi et al. 1978). Therefore, we first investigated whether methanol was consumed by $\mathrm{mADH}$ using both the parental and adhA-deficient mutant $(\triangle a d h A)$ strains of $G$. oxydans. Both strains were cultured in glucose medium for $24 \mathrm{~h}$ and then inoculated into glycerol medium containing up to $10 \%(v / v)$ methanol. As shown in Figure 4, no significant differences in methanol concentration in the culture broth were observed between the parental and $\triangle a d h A$ strains of $G$. oxydans for up to $10 \mathrm{~h}$, indicating that $\mathrm{mADH}$ is not directly involved in methanol oxidation. In addition, methanol supplementation to the $\triangle a d h A$ strain did not significantly alter the production of DHA (Figure 4).

Next, we measured glycerol dehydrogenase activity in the presence of methanol using purified mADH. Glycerol dehydrogenase activity in the absence of methanol increased as the glycerol concentration increased, reaching $5.9 \mathrm{U} / \mathrm{mg}$ at $20 \%(w / v)$ glycerol. At this concentration, a rapid decrease in dehydrogenase activity with increasing methanol concentration was observed (Figure 5). In the presence of $2 \%(w / v)$ methanol, glycerol dehydrogenase activity decreased to one tenth that in the absence of methanol. The half-maximal inhibitory concentration $\left(\mathrm{IC}_{50}\right)$ of methanol was estimated to be $\sim 0.31 \%(w / v)$. These results suggest that $<1 \%(w / v)$ methanol inhibited $\mathrm{mADH}$ catalyzed oxidation at a high concentration of glycerol.

\section{Discussion}

Our data indicate that GA production by G. frateurii NBRC103465 decreased when the microbe was cultured using raw glycerol (Figure 2) and a synthetic solution modeled after the raw glycerol, which contains $30 \%(w / v)$ methanol (Figure 3), for $\mathrm{pH}$ control. This phenomenon also occurred in flask cultures of other Gluconobacter spp. (Table 1). Thus, methanol was likely the cause of the decrease in GA production.

Previously, we showed that $\mathrm{mADH}$ is a key enzyme in the production of GA from glycerol, and that its deletion 
Table 1 Effect of methanol supplementation on GA and DHA production by Gluconobacter spp.

\begin{tabular}{ccccccr}
\hline & \multicolumn{2}{c}{ G. frateurii NBRC103465 } & \multicolumn{2}{c}{ G. frateurii THD32 } & \multicolumn{2}{c}{ G. oxydans IFO12528 } \\
\hline Initial methanol $(\%, v / v)$ & 0 & 1 & 0 & 1 & 0 & 1 \\
GA $(\mathrm{g} / \mathrm{L})$ & $28.2 \pm 0.3$ & $14.4 \pm 0.7$ & $23.0 \pm 0.3$ & $6.8 \pm 0.3$ & $18.8 \pm 1.0$ & $5.9 \pm 0.4$ \\
DHA $(\mathrm{g} / \mathrm{L})$ & $15.9 \pm 0.6$ & $38.0 \pm 1.7$ & $25.1 \pm 5.8$ & $24.1 \pm 1.4$ & $33.7 \pm 0.3$ & $51.9 \pm 2.1$ \\
\hline
\end{tabular}

Cells were cultivated in $30 \mathrm{~mL}$ of glycerol medium containing $170 \mathrm{~g} / \mathrm{L}$ glycerol with methanol supplementation at $30^{\circ} \mathrm{C}$ for $96 \mathrm{~h}$. The averages and standard deviations of two independent experiments are presented.

from G. oxydans $(\triangle a d h A)$ resulted in a strain incapable of producing GA (Habe et al. 2009a). In addition, mADH exhibits higher dehydrogenase activity toward methanol than toward glycerol (Adachi et al. 1978). Therefore, we hypothesized that mADH catalyzed the oxidation of methanol more easily than the oxidation of glycerol, resulting in a decrease in the methanol consumption rate in the broth of the $\triangle a d h A$ mutant. However, no differences in methanol consumption between the parental and $\triangle a d h A$ strains of G. oxydans grown in glycerol containing up to $10 \%(v / v)$ methanol were detected (Figure 4). Next, the inhibitory effect of methanol on glycerol oxidation by $\mathrm{mADH}$ was evaluated using purified $\mathrm{mADH}$. A comparison of mADH activity at high glycerol concentrations with or without methanol demonstrated that methanol inhibited glycerol dehydrogenase activity (Figure 5). This suggests that methanol decreased the rate of glycerol oxidation by $\mathrm{mADH}$, resulting in reduced GA production. Activity of $\mathrm{mADH}$ toward various amount of glycerol (5, $10,15$, and $20 \%, w / v)$ in the presence of methanol $(0.3 \%$,

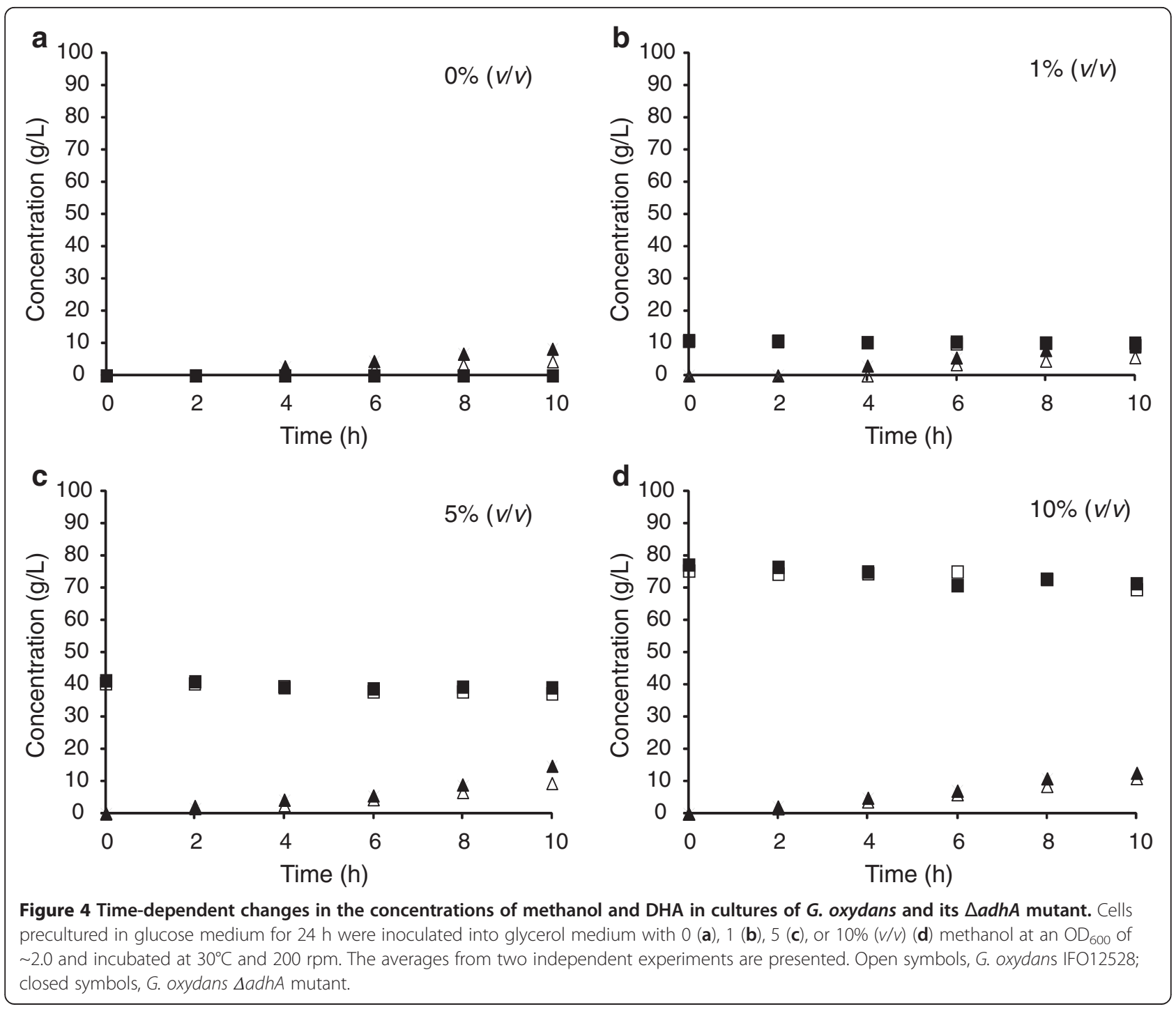




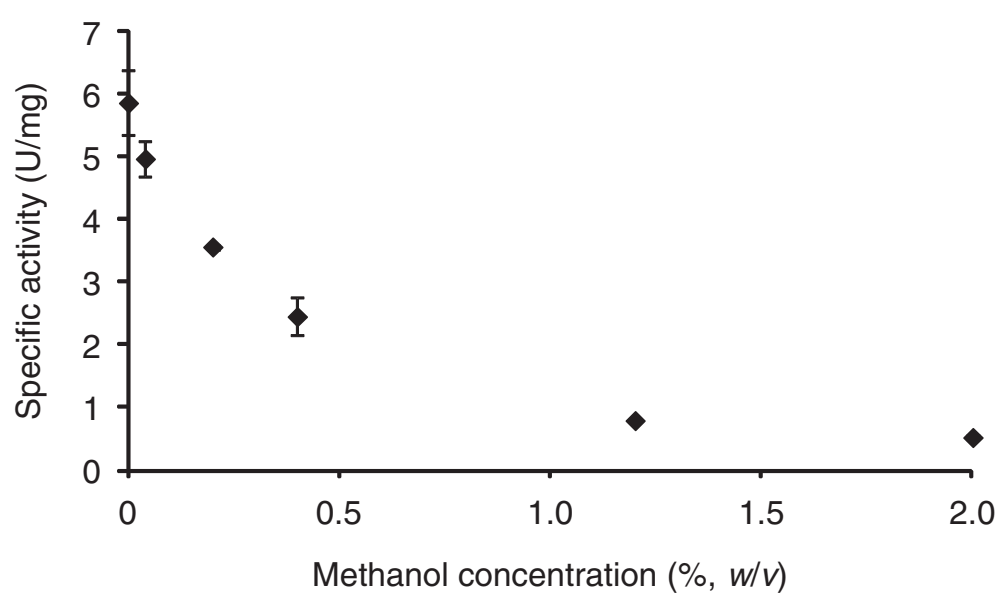

Figure $\mathbf{5}$ Methanol inhibition of glycerol oxidation by $\mathbf{m A D H}$. The specific activity toward $20 \%(\mathrm{~W} / \mathrm{V})$ glycerol was measured using potassium ferricyanide as the electron acceptor. The data are presented as the averages and standard deviations from three independent experiments.

$w / v)$ was also measured. A gradual increase in dehydrogenase activity was observed as the glycerol concentration increased, although methanol inhibited dehydrogenase activity constantly by approximately $60 \%$ as compared to that in the absence of methanol (data not shown). This could suggest noncompetitive inhibition by methanol in mADH glycerol dehydrogenation; however, detailed kinetic analyses of mADH activity toward glycerol and/or methanol will be necessary to clarify the mechanism whereby methanol inhibits the oxidation of glycerol by $\mathrm{mADH}$. In addition, fed-batch cultivation with a raw glycerol model solution (Figure 3 ) revealed that the methanol concentration in the broth reached 0.4 and $1.5 \%$ after 12 and $18 \mathrm{~h}$ of cultivation, respectively (data not shown). Considering the $\mathrm{IC}_{50}$ value for methanol in the oxidation of glycerol by $\mathrm{mADH}, \mathrm{GA}$ production was probably inhibited even in the early stages of cultivation.

Because the $\triangle a d h A$ strain of G. oxydans showed similar DHA production profiles in the presence of methanol as well as the parental strain (Figure 4), methanol was supposed to enhance DHA production regardless of GA production. In contrast, our preliminarily experiment showed that a membrane-bound glycerol dehydrogenase (SldAB)-defective mutant of G. frateurii THD32 ( $\Delta$ sldA), which is incapable of producing DHA, showed a clear decrease in GA production in the presence of $1 \%$ methanol (data not shown), as well as the parental strain G. frateurii THD32. This suggests that methanol inhibited GA production regardless of DHA production. These complementary experiments imply that methanol would have an influence on activities of individual enzymes involved in each production. In contrast, DHA production by $G$. frateurii THD32 was not significantly changed in the presence of $1 \%$ methanol (Table 1). Hence, studies on cell responses at the transcriptomic and metabolomic levels in the presence of methanol will help elucidate the detailed mechanism of methanol-related DHA production and the difference among Gluconobacter strains in effect of methanol on DHA production.

Gluconobacter spp. tend to produce both GA and DHA at a high concentration (170 g/L) of glycerol (Table 1). In terms of DHA production, this is a problem; that is, a high concentration of glycerol during DHA fermentation results in increased byproduct (GA) formation (Figure 1). In addition, a higher initial concentration of glycerol decreased the rates of cell growth and DHA production (Claret et al. 1992). Therefore, attempts have been made to improve DHA production by process engineering $(\mathrm{Hu}$ et al. 2011) and strain development (Habe et al. 2010). However, our data show that G. oxydans, an industrial strain used for DHA production, produced a large quantity of DHA from $170 \mathrm{~g} / \mathrm{L}$ glycerol with less GA production in the presence of $1 \%$ methanol (Table 1). This indicates that efficient DHA production by G. oxydans using a high concentration of glycerol can be achieved by adding a small amount of methanol to the culture. In this case, methanol seemed to act not only as an inhibitor of GA formation, but also as an enhancer of DHA formation. This suggests that BDF-derived raw glycerol containing methanol would be a good source material for efficient and economical DHA production with less byproduction of GA, although a clear mechanism for enhancing DHA production is unclear.

In summary, GA production in the presence of methanol decreased with the production of DHA by Gluconobacter spp. The dehydrogenase activity of $G$. oxydans mADH toward glycerol was inhibited by methanol, suggesting that the rate of glycerol oxidation catalyzed by mADH determines the product selectivity of Gluconobacter spp. between GA and DHA. Recombinant $G$. frateurii with an enhanced ability to assimilate methanol or mADHs with a lower affinity for methanol 
will be necessary for the efficient production of GA from raw glycerol.

\section{Competing interests}

The authors declare that they have no competing interests.

\section{Authors' contributions}

$H H, T Y, N M, D K$, and KM designed research; SS, HH, and TY performed research; SS, HH, and TY analyzed data; and SS and HH wrote the paper. All authors read and approved the final manuscript.

\section{Acknowledgements}

This work was financially supported in part by the Japan-US Cooperation Project for Research and Standardization of Clean Energy Technologies from the Ministry of Economy, Trade, and Industry of Japan.

\section{Author details}

${ }^{1}$ Research Institute for Innovation in Sustainable Chemistry, National Institute of Advanced Industrial Science and Technology (AIST), Tsukuba Central 5-2, 1-1-1 Higashi, Tsukuba, Ibaraki 305-8565, Japan. ${ }^{2}$ Bioproduction Research Institute, National Institute of Advanced Industrial Science and Technology (AIST), 2-17-2-1 Tsukisamu-higashi, Toyohira-ku, Sapporo 062-8517, Japan. ${ }^{3}$ Department of Biological Chemistry, Faculty of Agriculture, Yamaguchi University, 1677-1 Yoshida, Yamaguchi 753-8515, Japan.

Received: 18 March 2013 Accepted: 20 March 2013 Published: 2 April 2013

\section{References}

Adachi O, Tayama K, Shinagawa E, Matsushita K, Ameyama M (1978) Purification and characterization of particulate alcohol dehydrogenase from Gluconobacter suboxydans. Agric Biol Chem 42:2045-2056

Claret C, Bories A, Soucaille P (1992) Glycerol inhibition of growth and dihydroxyacetone production by Gluconobacter oxydans. Curr Microbiol 25:149-155

Eriksson CJP, Saarenmaa TPS, Bykov IL, Heino PU (2007) Acceleration of ethanol and acetaldehyde oxidation by D-glycerate in rats. Metabolism 56:895-898

Fukuoka T, Habe H, Kitamoto D, Sakaki K (2011) Bioprocessing of glycerol into glyceric acid for use in bioplastic monomer. J Oleo Sci 60:369-373

Fukuoka T, Ikeda S, Habe H, Sato S, Sakai H, Abe M, Kitamoto D, Sakaki K (2012) Synthesis and interfacial properties of monoacyl glyceric acid as a new class of green surfactants. J Oleo Sci 61:343-348

Glycerin Market Report (2011) Oleoline., p 94

Habe H, Shimada Y, Yakushi T, Hattori H, Ano Y, Fukuoka T, Kitamoto D, Itagaki M, Watanabe K, Yanagishita H, Matsuhita K, Sakaki K (2009a) Microbial production of glyceric acid, an organic acid that can be mass produced from glycerol. Appl Environ Microbiol 75:7760-7766

Habe H, Fukuoka T, Kitamoto D, Sakaki K (2009b) Biotechnological production of D-glyceric acid and its application. Appl Microbiol Biotechnol 84:445-452

Habe H, Fukuoka T, Kitamoto D, Sakaki K (2009c) Biotransformation of glycerol to D-glyceric acid by Acetobacter tropicalis. Appl Microbiol Biotechnol 81:1033-1039

Habe H, Shimada Y, Fukuoka T, Kitamoto D, Itagaki M, Watanabe K, Yanagishita H, Sakaki K (2009d) Production of glyceric acid by Gluconobacter sp. NBRC3259 using raw glycerol. Biosci Biotechnol Biochem 73:1799-1805

Habe H, Fukuoka T, Morita T, Kitamoto D, Yakushi T, Matsushita K, Sakaki K (2010) Disruption of the membrane-bound alcohol dehydrogenase-encoding gene improved glycerol use and dihydroxyacetone productivity in Gluconobacter oxydans. Biosci Biotechnol Biochem 74:1391-1395

Habe H, Sato S, Fukuoka T, Kitamoto D, Sakaki K (2011a) Effect of glyceric acid calcium salt on the viability of ethanol-dosed gastric cells. J Oleo Sci 60:585-590

Habe H, Fukuoka T, Sato S, Kitamoto D, Sakaki K (2011 b) Synthesis and evaluation of dioleoyl glyceric acids showing antitrypsin activity. J Oleo Sci 60:327-331

Hu ZC, Zheng YG, Shen YC (2011) Use of glycerol for producing 1,3dihydroxyacetone by Gluconobacter oxydans in an aillift bioreactor. Bioresour Technol 102:7177-7182

Kubicek P, Sladek P, Buricova I (2005) Method of preparing dichloropropanols from glycerine., WO2005/021476
Rahmat N, Abdullah AZ, Mohamed AR (2010) Recent progress on innovative and potential technologies for glycerol transformation into fuel additives: A critical review. Renew Sustain Energy Rev 14:987-1000

Rathnasingh C, Raj SM, Jo JE, Park S (2009) Development and evaluation of efficient recombinant Escherichia coli strains for the production of 3hydroxypropionic acid from glycerol. Biotechnol Bioeng 104:729-739

Rehman A, Wijesekara SRG, Nomura N, Sato S, Matsumura M (2008) Pretreatment and utilization of raw glycerol from sunflower oil biodiesel for growth and 1,3-propanediol production by Clostridium butyricum. J Chem Technol Biotechnol 83:1072-1080

Soemphol W, Adachi O, Matsushita K, Toyama H (2008) Distinct physiological roles of two membrane-bound dehydrogenases responsible for D-sorbitol oxidation in Gluconobacter frateurii. Biosci Biotechnol Biochem 72:842-850

Toyama H, Soemphol W, Moonmangmee D, Adachi O, Matsushita K (2005) Molecular properties of membrane-bound FAD-containing D-sorbitol dehydrogenase from thermotolerant Gluconobacter frateurii isolated from Thailand. Biosci Biotechnol Biochem 69:1120-1129

Wood WA, Fetting RA, Hertlein BC (1962) Gluconic dehydrogenase from Pseudomonas fluorescens. Methods Enzymol 5:287-291

doi:10.1186/2191-0855-3-20

Cite this article as: Sato et al:: Change in product selectivity during the production of glyceric acid from glycerol by Gluconobacter strains in the presence of methanol. AMB Express 2013 3:20.

\section{Submit your manuscript to a SpringerOpen ${ }^{\circ}$ journal and benefit from:}

- Convenient online submission

- Rigorous peer review

- Immediate publication on acceptance

- Open access: articles freely available online

High visibility within the field

- Retaining the copyright to your article

Submit your next manuscript at $\gg$ springeropen.com 